\title{
Enhanced Performance of Developed Two-Step (Hybrid/PMU) Linear State Estimator Model
}

\author{
Shaymaa M. Khamis and Nabil H. Abbasy
}

\begin{abstract}
This paper proposes a novel hybrid state estimation method. In traditional EMS (Energy Management System), the ordinary hybrid state estimation technique employs both conventional measurements from the SCADA (Supervisory Control and Data Acquisition) and PMU (Phasor Measurements Unit) measurements in the state estimation software. Alternatively PMU measurements can be added through a post-processing or pre-processing step with the results of traditional state estimator to obtain the final estimation results. While this paper presents a new two-step (hybrid/PMU) algorithm where the first step adopts a hybrid state estimation SCADA/PMU and the second step is a linear state estimation comprises PMU measurements and the state vector resulting from the first step; consequently a double hybridization is achieved. Alexandria $(330 \mathrm{~km}$ north Cairo, Egypt) 22 -bus system is used as case study. The simulation results demonstrate that the proposed algorithm enhances the precision and accuracy of state estimation greatly because the PMU measurements hybridize in two successively steps. Better dynamic behavior as compared with other state estimators is obtained.
\end{abstract}

Index Terms-Hybrid state estimation, linear state estimation, observability, phasor measurement unit, state estimation, supervisory control and data acquisition.

\section{INTRODUCTION}

Since the beginning in the 1960's, the power system network has been growing larger and more complex; operators used to collect a set of incomplete and unsynchronized measurements and inserted them into a load flow calculation in an attempt to find the operating point of the system [1]. This was a very inexact approach and in most times led to divergence of the load flow equations. In 1970, Schweppe and Wildes suggested new methods of implementing state estimation in power systems [2]. Then the analysis of real time data received from RTU (Remote Terminal Units) in the SCADA (Supervisory Control and Data Acquisition) system is possible, more accurate and extensive but it was difficult to get the voltage phase angle because time reference is not synchronized. Synchronized PMU (Phasor Measurement units) were launched in mid1980s. These digital metering devices are used in conjunction with a timed GPS (Global Positioning System) signal to provide synchronized phasor measurements at different locations in the power system. With GPS synchronization, measurements are synchronized regardless

Manuscript received September 28, 2018; revised May 1, 2019.

Shaymaa M. Khamis is with Egyptian Electricity Transmission Company, Alexandria, Egypt (e-mail: eng.chaymaa@gmail.com).

Nabil H. Abbasy is with Electrical Department, Faculty of Engineering, Alexandria, Egypt (e-mail: abbasyna@ hotmail.com). the large distances which may separate metering points and the magnitude and phase angle of voltages and currents are determined accurately [3]. In 1992, early prototypes of the PMU were built, and Macrodyne constructed the first PMU (model 1690). In the latter years, PMUs were continuously inserted in the monitoring and control of power systems. Consequently the PMU is used in distributed dynamic state estimator on the widest range. PMUs are used to measure voltages, current, angle and frequency, and can supply realtime information about the state of large portions of grids and allow one to obtain a true snapshot of the power system, making the technology for real-time supervision of power systems clearly at hand [4]. A model of new two-step hybrid state estimation using SCADA and PMU measurements is discussed in this paper. This model is implemented in Alexandria 22-bus system for enhancing the state estimation and gets better dynamic behavior.

This paper is organized as follow: a review about the traditional state estimation, state estimator using PMU only, the hybrid SCADA/PMU and their algorithms is presented in section II. The new proposed two-step (hybrid/ PMU) is proposed in section III. Section IV shows the case study model structure used to simulate the proposed algorithm. The simulation results carried out on Alexandria $220 \mathrm{kV}$ power system are shown in section $\mathrm{V}$, and are followed by conclusion in section VI.

\section{STATE Estimation}

\section{A. The SCADA-Oriented State Estimator (SOSE)}

This is a high speed state estimator which resides in the SCADA subsystem and was fast enough to execute, ideally, power system state estimation uses a SCADA system to collect the real-time measurement data, which involves injection measurements, line flow measurements and voltage magnitude measurements and every data acquisition scan [5],[6]. The State estimation collects data from SCADA operations model and topology, detects bad measurements and estimates a new state after that returns them to SCADA model [7]. It provides complete and more reliable information to other advanced applications; e.g. the Intelligent Alarm Processor (IAP), contingency Analysis (CA) and the Automatic Generation Control (AGC). The SOSE is expected to be a static estimator that executes each 4 - 10 seconds periodically. However, when the system state changes during the scan, the systems dynamics can be ignored at such time periods. The data retrieved no longer represents the system state accurately. The incorrect and inaccurate data of the SCADA system lead to deviation between the power system real values and the sampling data calculated at time frames of simulation [8]. 


\section{B. State Estimator Using PMU Only}

Regarding state estimation, Measurements received from PMUs are more accurate with small variances compared to the variances of conventional measurements. Therefore, inserting more PMU measurements is preferred to produce more accurate estimates [9].It is known that, the PMUs of WAMS (Wide Area Measurement System) are not sufficient for static state estimation in most of the existing power systems because of the high price [10].An ultimate target is to populate power systems with enough PMUs to provide full observability, where sufficient number of PMUs is available to carry out state estimation exclusively using PMUs and disregarding all the conventional measurements, due to the relatively higher cost of PMUs and the requirement of the associated communication and data management facilities. However, the feasibility of a PMUonly measurement system is still a distant possibility in most power systems and this will still be a few years away.

\section{State Estimator Using Hybrid SCADA/PMU}

With the growing number of PMU's, it is anticipated that a number of utilities use the PMUs in an incremental fashion, in conjunction with conventional measurements for the state estimation of power system in particular mixing algorithm methods [11].The accuracy of the PMU measurements is much higher than the conventional measurements; the phasor technology frame time' sub-second data is typically 20,30 or 60 samples/second. "Fig. 1" shows the contribution of PMU in SCADA. Phasor technology provides the full observability of dynamic behavior of power systems which can overcome the weakness of the traditional SCADA-based systems. It provides the magnitude and angle of the voltage and current very easily. Many comparisons are established between different estimation techniques used the phasor measurement units (PMUs) under both normal and transient conditions to analyze the efficiency and robustness of hybrid state estimation [12], [13].

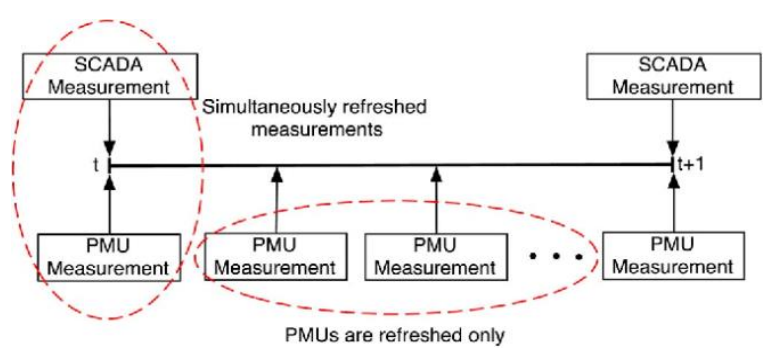

Fig. 1. The Contribution of PMU in SCADA

Several state estimation methods attempted to tackle the problem of incomplete PMU coverage and the inaccurate SCADA results through a two-stage verification method. For example, Simen Karlsen [14] proposed a two-pass method. The first pass utilizes classical static state estimation through WLS using the system's SCADA collected measurements; the second pass is a linear state estimation using PMU measurements as well as the state vector resulting from the first pass. Nikolaos M. Manousakis, George N. Korres [15] proposed a two stage method. A Two-Level Power System Linear State Estimator is provided by T. Yang [16]. The previous classification of state estimation discussed the type of measurements, their accuracy and their scan times whereas the algorithm of PMU based state estimation can be classified into:

\section{Non-linear WLS Estimator}

Measurements are mainly coming from the SCADA systems. The measurements to be used include the bus voltage magnitude, the branch active and reactive power flows for a line or transformer, and the bus active and reactive power injections. The output of the estimation algorithm is state vector $x$ which consists of the voltage magnitudes $\mathrm{V}$ and phase angles $\theta$ at all buses except one bus. This bus is taken as a reference for the phase angles. The system measurement equation is:

$$
Z=h(x)+v
$$

where:

$z$ is the $(m \times 1)$ measurement vector; $x$ is the $(n \times 1)$ state vector to be estimated;

$h$ is a vector of nonlinear functions that relate the states to the measurements;

$v$ is an $(m \times 1)$ measurement error vector. It is necessary that $m \geq n$ and the Jacobian matrix of $h(x)$ has rank $n$ [17]

Linear WLS Estimator

The function $h(x)$ becomes linear when $[\mathrm{H}]$ is a constant matrix $(m \times n)$. It requires one iteration only to converge [18]. The states are characterized by the real and imaginary parts of rectangular bus voltages form for each bus in the network. Measurements are gathered from two data sources: the group of PMU devices and the other is the set of pseudo measurements derived from branch power flow measurements of SCADA systems [19].

\section{PROPOSED HYBRID TWO-STEP STATE ESTIMATION}

In this paper, a new 2-step hybrid SE algorithm is proposed, where the first step adopts a hybrid state estimation SCADA/PMU which is linear state estimation(LSE)based on Kaushik Das model [20] and the second step is a linear state estimation comprises PMU measurements as well as the state vector resulting from the first step.

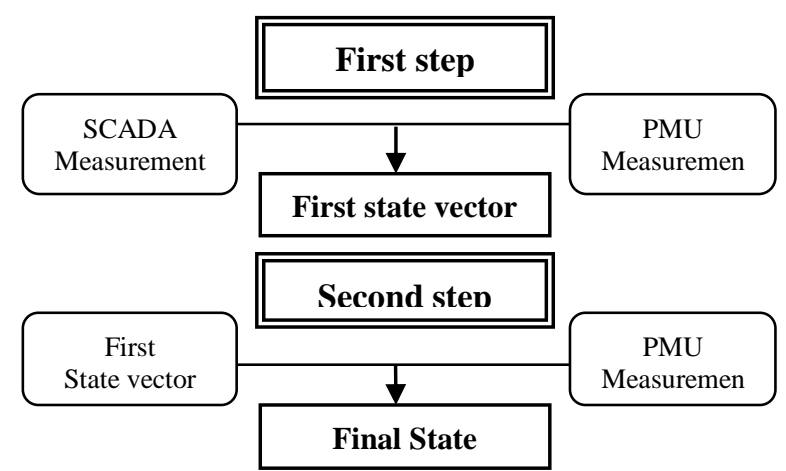

Fig. 2. Block diagram of proposed hybrid two-step state estimation.

The first step is a hybrid state estimation combines conventional SCADA pseudo measurements' set of line parameters, bus voltage and limited PMU measurements (synchronized voltage magnitude and angle) in an interpolation algorithm. If measurements from PMU or 
SCADA change significantly, the pre-computed interpolation matrix $(\mathrm{H})$ is modified by a sensitivity change matrix $(\Delta \mathrm{H})$. The modified matrix tracks the system variation more accurately. The second step is a linear state estimation using PMU measurements and the state vector resulting from the first step in a non iterative weight least square model. "Fig. 2" shows the block diagram of proposed Hybrid Linear State Estimation

\section{A. The First Step Mathematics Algorithm}

The node voltage equation representing the electric circuit can be presented as:

$$
\left[I_{B U S}\right]=\left[Y_{B U S}\right] .\left[E_{B U S}\right]
$$

where,

$I_{B U S}$ : bus injection current vector, $Y_{B U S}$ : bus admittance matrix, $\mathrm{E}_{\mathrm{BUs}}$ : node voltage vector.

The bus admittance matrix $\mathrm{Y}_{\mathrm{BUS}}$ is partitioned into four sub-matrices:

$Y_{o o}:$ self admittance of observable buses, $Y_{u и}$ : self admittance of unobservable buses.

$Y_{\text {оu }}, Y_{\text {иo: }}$ the mutual admittances between observable and unobservable buses .

The nodal equation becomes as follows:

$$
\left[\begin{array}{l}
I o \\
I u
\end{array}\right]=\left[\begin{array}{ll}
Y o o & Y o u \\
Y \text { uo } & Y u u
\end{array}\right]\left[\begin{array}{l}
E o \\
E u
\end{array}\right]
$$

$I_{o}, E_{o}$ : the observed current and voltage through PMU

$I_{u}, E_{u}$ : the unobserved current and voltage through PMU, $\mathrm{N}_{\mathrm{u}}$ : number of unobserved buses

The injections $I_{U}$ at the unobserved buses are equivalent to load admittances.

$$
\left[I_{U}\right]=\left[\left(P_{i}-j Q_{i}\right) / E_{i}\right]
$$

where: $i=1,2, \ldots ., N_{U}$,

$P_{i}$ : injected active power in bus $i, Q_{i}$ : injected reactive power in bus $i$

$$
\left[Y_{U}\right]=\left[Y_{L}\right]=\left[\left(P_{i}-j Q_{i}\right) /|E i|^{2}\right]
$$

Equation (3) now becomes:

$$
\left[\begin{array}{c}
\mathrm{Io} \\
0
\end{array}\right]=\left[\begin{array}{cc}
\text { Yoo } & \text { You } \\
\text { Yuo } & \text { YT }
\end{array}\right]\left[\begin{array}{c}
\text { Eo } \\
\mathrm{Eu}
\end{array}\right]
$$

where,

$$
Y_{T}=Y_{U U}+\mathrm{Y}_{\mathrm{L}}
$$

Then

$$
\begin{gathered}
0=Y_{U O} \cdot E_{O}+Y_{T} \cdot \mathrm{E}_{\mathrm{U}} \\
E_{U}=-Y^{-1} T \cdot Y_{U O} \cdot E_{O}
\end{gathered}
$$

Yielding the interpolation model of unobserved buses in matrix form

$$
E_{U}=H \cdot E_{O}
$$

If power flow changes in between two successive PMU measurements, a method of updating the interpolators $H$ is: where

$$
\Delta \mathrm{H}=-\left(Y_{T}^{0}\right)^{-1} \cdot\left(\operatorname{diag}\left[S_{u}^{\prime} /\left|E_{u}^{\prime}\right|^{2}-S_{u}^{0} /\left|E_{u}^{0}\right|^{2}\right]\right) \cdot H
$$

$S_{u}^{\prime}$ : the power of unobservable buses at the present configuration,

$S^{0}{ }_{u}$ : the power of unobservable buses at the initial operating configuration,

$E_{u}^{\prime}$ : is the voltage of unobservable buses at the present configuration,

$E_{u}^{0}$ : is the voltage of unobservable buses at the initial operating configuration. Hence, the interpolation formula for unobserved buses tracking system dynamics during fault or sudden change in load becomes:

$$
E_{U}=(H+\Delta H) \cdot E_{O}
$$

\section{B. The Second Step Mathematics Algorithm}

The second step linear estimation is performed mathematically where the state vector from the first step, referred to as the Interpolated state estimation vector (ISE), comes and is treated as a set of measurements, and the other set of measurements are Synchrophasor measurements of bus voltages and line currents in rectangular coordinates, along with their physical locations in the network [20]. The complete measurement model presented in "(13)" is expanded to facilitate the incorporation of the Interpolated estimated state vector as shown in "(14)" below. The subscripts (ISE) and (PMU) refer to values obtained from the Interpolated state estimator's state vector and PMU measurements, respectively.

$$
Z=H \cdot x+\varepsilon
$$

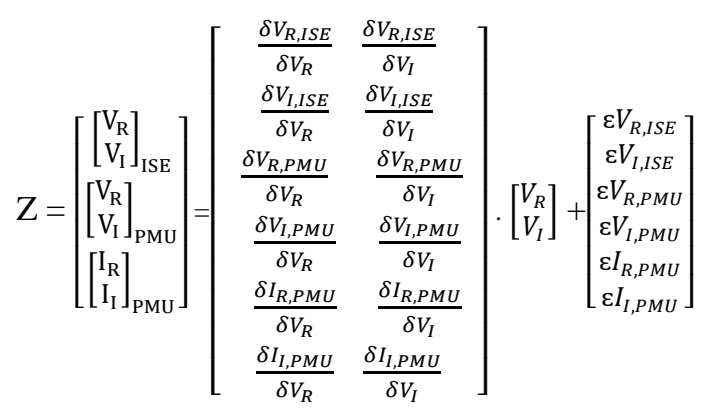

\section{MODEL STRUCTURE}

The proposed 2-step hybrid SE algorithm is constructed as a set of Matlab scripts. It has been tested on 22-bus Alexandria network (300km north Cairo) $220 \mathrm{kV}$ transmission power system. In order to demonstrate the effectiveness of the proposed method, the proposed two-step method estimation results is compared with Kaushik Das' estimation method [20], the weighted least square (WLS) results [21] and with the true value obtained from time domain transient stability solution. A single-line diagram of the network is provided in "Fig. 3". It is a 22-bus system; There are two generation plant: SidiKrir and Abukir1,2 power stations. Kafr Eldwar, Abis, Suif, Etay, Smouha, Freezone, Karmouz, Somid, etc... substations. This system contains five PMUs, capable of voltage and line current measurements of connected branches. The system is 
completely observable through SCADA; real time data was retrieved from Alexandria Regional Control Center (ARCC).Whereas PMU data were generated using the time domain transient stability program "Power System Analysis Toolbox (PSAT)" [22].

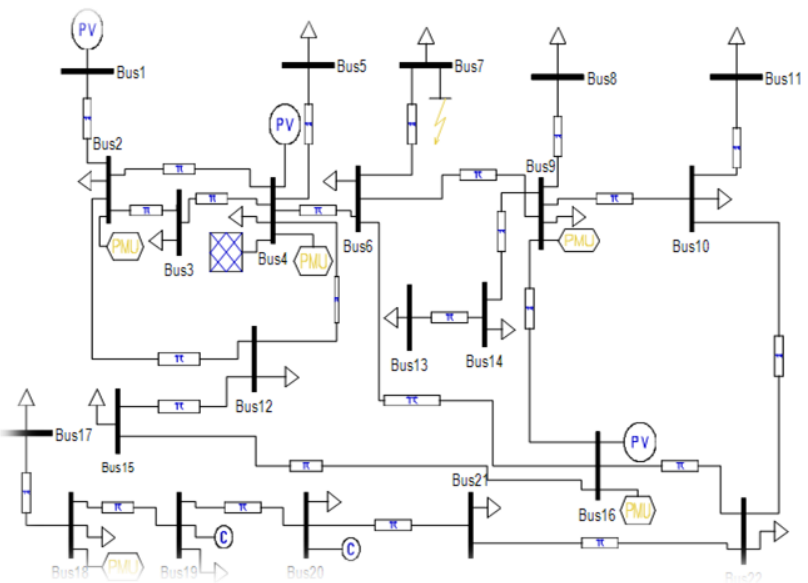

Fig. 3. 22-Bus alexandria network $220 \mathrm{Kv}$ transmission system.

The optimal PMU placement (OPP) fixed a little set of PMUs such that the major part of system is observable [8]. Five PMUs were placed at buses 2, 4, 9, 16, and 18 as shown in Figure (3), installed PMUs at buses make direct synchronized measurements of the voltage phasor and the current phasors of some or all the branches attached to the buses. By this (OPP) placement only 5 buses are unobservable through PMUs: 7, 11, 13, 20, and 21 as shown in "fig. 3".

The unobservable buses are interpolated from the observable buses by the interpolation matrix $(\mathrm{H})$. A sensitivity change matrix $(\Delta \mathrm{H})$ is computed to compensate the interpolation matrix, by this method system dynamics during fault and sudden change in load can be tracked. The state vector interpolated from the first step (ISE) state vector is treated in the second step as a set of measurements matrix, in addition to the PMU bus voltage measurements set and the PMU line current measurements set. All these measurements are used in a non-iterative weighted least square model. The SCADA supply the second step by information regarding the network topology through a complete list of the branches in the system, the buses connected by the branch, the series impedance and the total shunt admittance of the branch as a complex number.

\section{SimUlation RESUltS}

In this section we simulate the performance of the proposed algorithm using the 22-bus system as shown in "Fig. 3". The simulation is implemented in Matlab environment with the initial measurements (true values). WLS state estimation Matlab program and also kaushik Das method Matlab program are run to obtain their result values. In order to study the effectiveness of the proposed algorithm a set of results comparison is implemented with regard to the true value, WLS and kaushik Das results.

The bus voltage (magnitude and angle) over the 22-buses are plotted by using the three methods and compare them by the true value in "fig. 4, 5". From "Fig. 4", it is evident that the bus voltage magnitude in the proposed method is closer to the true value than of both Kaushik Das 'method and the WLS. On the other hand the bus voltage angle is enhanced greatly in the proposed than Kaushik Das but not better than the WLS method.

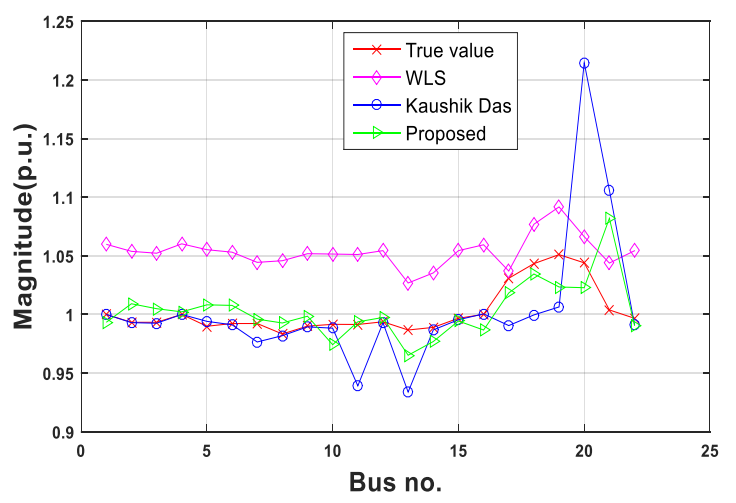

Fig. 4. Voltage magnitude comparison between WLS, Kaushik Das, proposed results and true values.

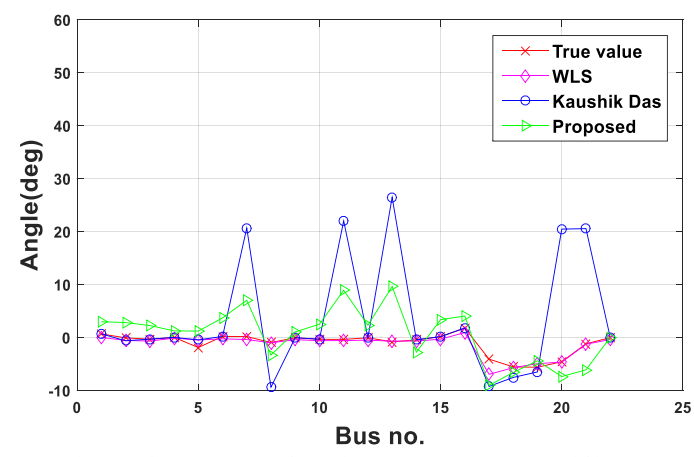

Fig. 5. Voltage phase comparison between WLS, Kaushik Das, proposed results and true values.

A time domain transient simulation is executed at "bus 13 "which is one of unobservable buses by using the WLS method, the Kaushik Das method, the proposed method and compared with the true value in "Fig. 6,7".

It is clear from "fig. 6" that proposed estimation voltage magnitude is much better than the other methods and is very much close to the actual. In "Fig. 7" the angle of proposed method is better than Kaushik Das method.

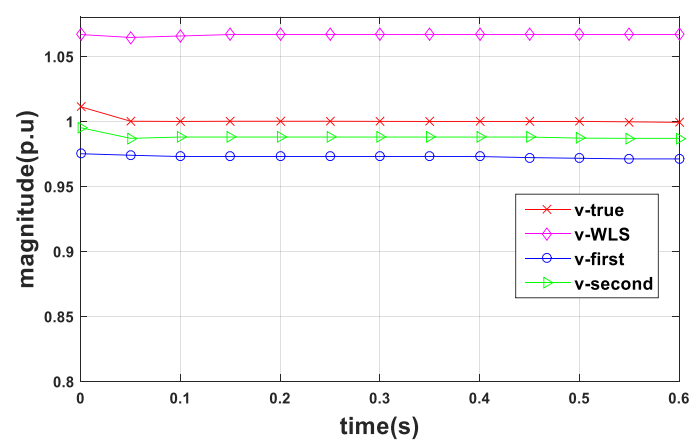

Fig. 6. Bus 13 Voltage magnitude comparison between WLS, Kaushik das, proposed results and true values.

A transient $(0.2 \mathrm{~s})$ three phase to ground fault is simulated at bus 7 which is unobservable through PMUs. By using the PSAT(Power System Analysis Toolbox) the fault is presented as in "fig. 3" and the time domain simulation is run for 1 second,the performance of the faulted bus 7 is presented in "Fig. 8, 9". The performance of bus 6 in the 
same time is displayed in "Fig. 10, 11".

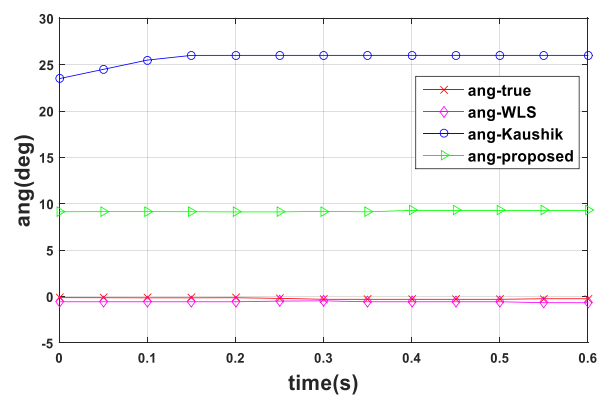

Fig. 7. Voltage angle at bus 13 comparison between WLS, Kaushik Das, proposed results and True values.

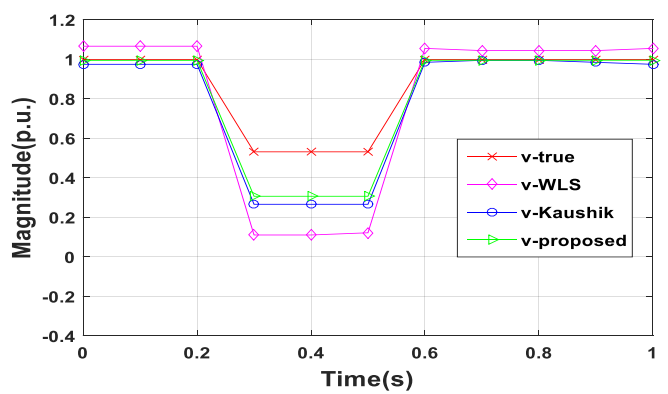

Fig. 8. Voltage magnitude for bus 7 at a three phase fault on bus 7 .

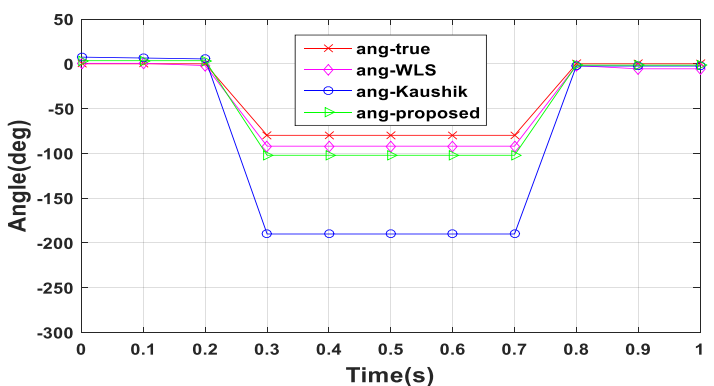

Fig. 9. Voltage angle for bus 7 at a three phase fault on bus 7.

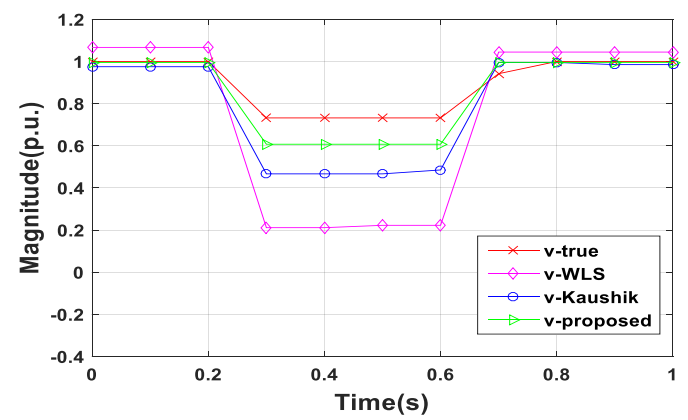

Fig. 10. Voltage magnitude of bus 6 for a three phase fault on bus7.

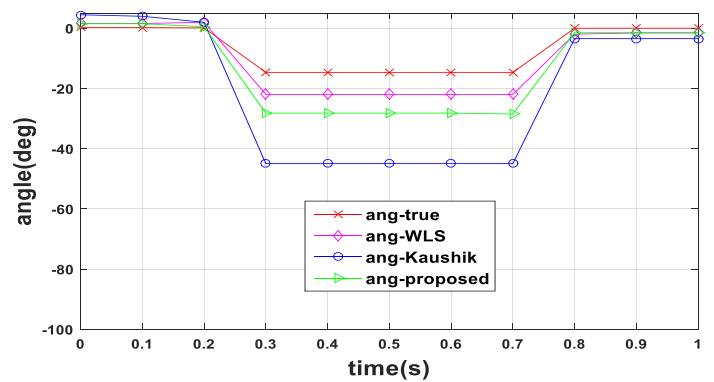

Fig. 11. Voltage angle of bus 6 for a three phase fault on bus 7 .

A standard deviation comparison between the voltage magnitude of proposed two-step method, Kaushik Das' method and the conventional WLS method is listed in table 1. It can be seen from the Table that the variance and standard deviation are the least in the proposed method, then the Kaushik Das then WLS method. "Fig. 12" shows the histogram graph for the three methods and the normal distribution.

TABLE I: STANDARD DEVIATION VolTAGE COMPARISON BETWEEN WLS,

\begin{tabular}{|c||c|c|c|}
\multicolumn{4}{|c|}{ KAUSHIK DAS AND PROPOSED METHODS } \\
\hline Buses & WLS'error & $\begin{array}{c}\text { Kaushik } \\
\text { Das'error }\end{array}$ & Proposed'error \\
\hline $\mathbf{1}$ & 0.1598 & 0 & 0.0067 \\
\hline$\vdots$ & $\vdots$ & $\vdots$ & $\vdots$ \\
\hline $\mathbf{2 2}$ & 0.15759 & 0.00531 & 0.00601 \\
\hline \hline Error average & 0.147546 & 0.024735455 & 0.014474545 \\
\hline$\sigma^{\mathbf{2} \text { (Variance) }}$ & 0.0004344 & 0.001703108 & 0.000239758 \\
\hline $\begin{array}{c}\text { Standard } \\
\text { deviation }\end{array}$ & 0.02084228 & 0.041268726 & 0.015484131 \\
\hline
\end{tabular}

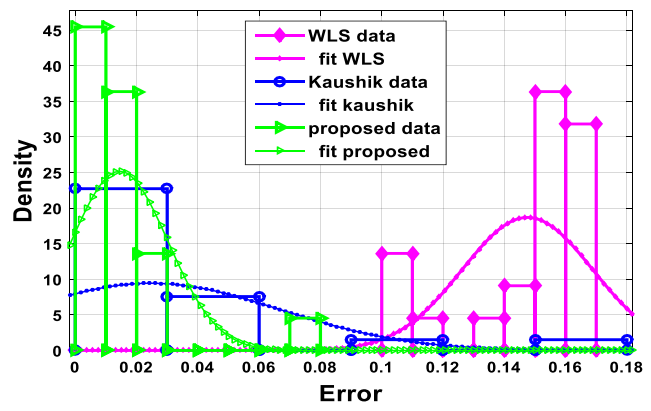

Fig. 12. Histogram graph and normal distribution for the error in three methods

It is clear from the histogram that the normal curve is shorter and broader in the Kaushik Das method which means that the variance increases in this method whereas in the proposed method the variance is small (where most values occur very close to the mean) because the curve is narrow and tall [23].

\section{CONCLUTION}

This paper presented a novel hybrid state estimation algorithm combining SCADA and PMU measurements in form of double hybridization successive steps in order to increase the accuracy and effectiveness. The simulation results presented clearly that the proposed state estimation technique could accurately track the system transient even in the presence of a disturbance in the unobservable bus in the system and with values more closed to the true values. Also results show that standard deviation and variance are the least in this proposed method.

\section{REFERENCES}

[1] A. G. Phadke and J. S. Thorp, "Synchronized phasor measurements and their application," Springer Science + Business Media, 2008.

[2] F. C. Schweppe and J. Wildes, "Power system static state estimation," IEEE Transactions on Power Apparatus and Systems, vol. PAS89,1970 .

[3] A. G. Phadke, "Synchronized phasor measurements - A historical overview," in Proc. Transmission and Distribution Conference and Exhibition 2002, vol. 1, pp. 476-479, 2002. 
[4] P. Kund and A. K. Pradhan, "Wide area measurement-based protection support during power swing," Int. J.Electr. Power Energy Syst, 2014, vol. 635, pp. 546-554.

[5] W. Zheng, W. Wu, and A. G. Exposito, "Distributed robust bilinear state estimation for power systems with nonlinear measurements," IEEE Trans. Power Syst., 2017, vol. 32, pp. 499-509.

[6] L. Hu, Z. Wang, and I. Rahman, "A constrained optimization approach to dynamic state estimation for power systems including PMU and missing measurements," IEEE Trans. Control Syst. Technol., 2016, vol. 24, pp. 703-710.

[7] A Roberts, T berry, and W. Wilson, "Modern distribution management system for regional electricity companies," Schneider Electric Ltd, UK.

[8] G. BeiBler, H. T. Neisius, and G. Schellstede "SCADA-oriented state estimation," IFAC Control of Power Systems and Power Plants, Beijing, China, 1997.

[9] Y. J. Yoon, "Study of the utilization and benefits of phasor measurement units for large scale power system state estimation," M.S. thesis, Electrical Engineering., Texas A\&M University, Texas 2005.

[10] S. S. M. Seyedi, F. Aminifar, and S. Afsharnia, "Parameter estimation of multiterminal transmission lines using joint PMU and SCADA,” IEEE Trans. Power Deliv., 2015, vol. 30, pp. 1077-1085.

[11] T. Jin and X. Y. Shen, "A mixed WLS power system state estimation method integrating a wide-area measurement system and Scada technology," Energies, 2018.

[12] T. Jiang, Y. Mu, and H. Jia, "A novel dominant mode estimation method for analyzing inter-area oscillation in china southern power grid," IEEE Trans. Smart Grid, pp. 2549-2560, 2016.

[13] J. Zhao, G. Zhang, and K. Das, "Power system real-time monitoring by using PMU-based robust state estimation method," IEEE Trans. Smart Grid, 2015, vol. 7, pp. 300-309.

[14] S. Karlsen, "Utilization of phasor measurement units in hybrid linear state estimation," M.S of Energy and Environmental Engineering, Dept. Elect. Eng., Norwegian Univ. Sci. Tech., January 2016

[15] N. M. Manousakis and G. N. Korres "A two-stage state estimator for power systems with PMU and SCADA measurements," School Elec. Comp. Eng., National Technical Univ., Athens, Greece.
[16] T. Yang, "Design of a two-level power system linear state estimator," Ph.D. Dissertation, Dept. Elect. Eng. and Comp. Sc., Washington State Univ., Washington, 2011.

[17] J. X. Chen, "State estimation and power flow analysis of power systems," Journal of Computers, vol. 7, no. 3, March 2012

[18] H. Sun, Z. Wang, and D. Nikovski, "Two-level state estimation method for power systems with SCADA and PMU measurements," Mitsubishi Electric Research Laboratories, May 2012.

[19] N. P. Theodorakatos and N. M. Manousakis, "Optimal placement of phasor measurement with linear and non-linear models," ECE Department, National Technical Univ. of Athens, Athens, Greece, 2015.

[20] K. Das and J. Hazra, "Real-time hybrid state estimation incorporating SCADA and PMU measurements," in Proc 3rd IEEE PES Innovative Smart Grid Technologies Europe (ISGT Europe), Berlin, 2012.

[21] R. F. Nuqui, "State estimation and voltage security monitoring using synchronized phasor measurements," Ph.D. dissertation, Virginia Polytechnic Institute and State University, July 2001.

[22] F. Milano, "Power system analysis toolbox," version 1.3.4, July 14, 2005

[23] M. J. D. Smith, "Statistical analysis handbook a comprehensive handbook of statistical concepts, techniques and software tools," 2018 Edition.

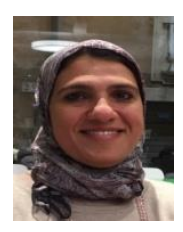

Shaymaa M. Khamis was bron in Egypt, in 1977.

She received her B.S in electrical engineering from Alexandria University, 2001,

She received her M.S in electrical engineering from Alexandria University, 2008

In 2002, she joined the Egyptian Electricity Transmission Company in Egypt as a control and data base engineer in Alexandria Regional Control Center, she has experience in substation automation, building of energy management systems, and control systems. she worked for nearly 15 years as design engineer in SCADA systems. 